\title{
Pulmonary adenocarcinoma appearing as ground-glass opacity nodules identified using non-enhanced and contrast-enhanced CT texture analysis: A retrospective analysis
}

\author{
JING YE* , JUN LING* , YAN LV, JUAN CHEN, JUNHUI CAI and MINGXIANG CHEN \\ Department of Medical Imaging, Yangzhou University Clinical College Subei People's Hospital, \\ Yangzhou, Jiangsu 225002, P.R. China
}

Received July 29, 2019; Accepted December 4, 2019

DOI: $10.3892 /$ etm.2020.8511

\begin{abstract}
The present study aimed to investigate the ability of CT-based texture analysis to differentiate invasive adenocarcinoma (IA) from pre-invasive lesions (PIL) or minimally IA (MIA) appearing as ground-glass opacity (GGO) nodules, and to further compare the performance of non-enhanced CT (NECT) images with that of contrast-enhanced CT (CECT) images. A total of 77 patients with GGO nodules and surgically confirmed pulmonary adenocarcinoma were included in the present retrospective study. Each GGO nodule was manually segmented and its texture features were extracted from NECT and CECT images using in-house developed software coded in MATLAB (MathWorks). The independent-samples t-test was used to select the texture features with statistically significant differences between IA and MIA/PIL. Multivariate logistic regression and receiver operating characteristics (ROC) curve analyses were performed to identify predictive features. Of the 77 GGO nodules, 12 were atypical adenomatous hyperplasia or adenocarcinoma in situ (15.6\%), 36 were MIA (46.8\%) and 29 were IA (37.7\%). IA and MIA/PIL exhibited significant differences in most histogram features and gray-level co-occurrence matrix features $(\mathrm{P}<0.05)$. Multivariate logistic regression and ROC curve
\end{abstract}

Correspondence to: Professor Yan Lv, Department of Medical Imaging, Yangzhou University Clinical College Subei People's Hospital, 98 Nan Tong West Road, Yangzhou, Jiangsu 225002, P.R. China

E-mail: 1vyan490183068@163.com

Abbreviations: IA, invasive adenocarcinoma; PIL, pre-invasive lesion; MIA, minimally invasive adenocarcinoma; GGO, ground-glass opacity; NECT, non-enhanced CT; CECT, contrast-enhanced CT; ROC, receiver operating characteristic; AIS, adenocarcinoma in situ; AUC, area under the curve; AAH, atypical adenomatous hyperplasia

*Contributed equally

Key words: diagnostic imaging, lung adenocarcinoma, solitary pulmonary nodule analyses revealed that smaller energy and higher entropy were significant differentiators of IA from MIA and PIL, irrespective of whether NECT images [area under the curve (AUC): 0.839, 0.859] or CECT images (AUC: 0.818, 0.820) are used. Texture analysis of CT images, regardless of whether NECT or CECT is used, has the potential to distinguish IA from PIL or MIA, particularly the parameters of energy and entropy. Furthermore, NECT images were simpler to obtain and no contrast agent was required; thus, analysis with NECT may be a preferred choice.

\section{Introduction}

Lung cancer is one of the most common tumor types worldwide and adenocarcinoma is the most common histological subtype. In 2011, the International Association for the Study of Lung Cancer, the American Thoracic Society and the European Respiratory Association proposed a novel international multidisciplinary classification system for lung adenocarcinoma, combined with the clinical, radiological, molecular biology and pathological characteristics of lung adenocarcinoma (1). Lung adenocarcinoma is divided into pre-invasive lesions (PIL), including atypical adenomatous hyperplasia (AAH) and adenocarcinoma in situ (AIS), as well as minimally invasive adenocarcinoma (MIA) and IA. The 5-year disease-free survival of patients with PIL and MIA is $100 \%$ after receiving segmentectomy of the lungs (2). However, for IA, lobectomy is the major method and the prognosis is relatively poor (3). Therefore, early detection and assessment of the invasiveness of lung adenocarcinoma are critical for the selection of surgical procedure and improving the prognosis (4).

Traditionally, pre-operative puncture biopsy and CT image interpretation have been used to judge the degree of infiltration of pulmonary adenocarcinoma appearing as ground-glass opacity (GGO) nodules. However, due to limitations of biopsy materials, it is difficult to accurately judge the invasiveness of the entire lesion. Using CT imaging, the invasiveness of the lesion is usually determined based on features including the size of the lesion, size and proportion of solid components, morphology, margin, internal features (vacuole sign, thickening of small vessels) and peripheral features (thoracic model traction and vascular aggregation). Studies have suggested 
that more solid components in GGO indicate more malignant invasion. Lee et al (5) suggested that the stretch of the thoracic model, size of the solid component and proportion of the solid component were all independent factors in differentiating invasive lung adenocarcinoma. In clinical application, differences exist in the understanding and recognition of CT features among radiologists of varying levels and qualifications. Until the last decade, it has been a challenge to differentiate the degree of infiltration of pulmonary adenocarcinoma through visual assessment of morphologic structures based on CT imaging due to considerable ambiguity between PIL, MIA and IA $(6,7)$.

A considerable number of lung adenocarcinomas appear as GGO nodules on CT images. When GGO nodules are small and represent as AAH or AIS, they grow along the alveolar walls only to appear as homogeneous GGO nodules (8). However, with the increase in invasive components in MIA and IA, the tumors may still appear as GGO nodules but may contain areas of regional voxel heterogeneity within the tumor. Thus, MIA and IA may still be regarded as a GGO nodule harboring a small central solid component measuring $5 \mathrm{~mm}$ or less or a pure GGO nodule $(9,10)$. Therefore, it was hypothesized that texture analysis and improved CT post-processing technology are feasible and valuable for the diagnosis, treatment monitoring and prognostic evaluation with several textural features, including skewness, kurtosis and entropy. They help detect the physical voxel-level changes within GGO nodules and may thus be used to distinguish IA from PIL or MIA. Non-enhanced CT (NECT) images as well as contrast-enhanced CT (CECT) images may be used for texture analysis. However, to the best of our knowledge, no previous study has investigated and confirmed which of the images are better.

Thus, the present study explored the value of texture analysis in distinguishing IA from PIL/MIA and investigated whether CT post-processing technology was better with NECT or CECT imaging.

\section{Materials and methods}

Patients. An experienced radiologist (Y.L.) retrospectively searched for patients between January 2015 and June 2018 including the time when they were first checked, using the descriptive terms 'GGO', 'GGN', 'ground-glass nodule' and 'ground-glass opacity' in the picture archiving and communication system of the Yangzhou University Clinical College Subei People's Hospital (Yangzhou, China) and 221 patients with 257 GGOs were retrieved. Another experienced radiologist (J.Y.) reviewed all of the CT scans. The further selection criteria were as follows: First, they must have undergone NECT and CECT scans with an interval time of no more than 3 months, and images must have been reconstructed with a thickness of $1.25 \mathrm{~mm}$. Furthermore, no operation or treatment, including biopsy, radiotherapy or chemotherapy, was performed prior to CT examination. Third, GGO nodules measured $\geq 5 \mathrm{~mm}$ and $\leq 3 \mathrm{~cm}$. In addition, the GGO nodules had none or little (regular, sharp) solid component. As an additional criterion, GGO nodules had no calcification, necrosis or cavitation, but a regular and sharp border. Furthermore, GGO nodules had no obvious malignant signs, including spiculation,
Table I. Patient characteristics $(n=77)$.

\begin{tabular}{lc}
\hline Characteristics & Value \\
\hline Age (years) & $53.40 \pm 11.13(23-75)$ \\
Sex (male/female) & $25 / 52$ \\
Pathologic subtype (mGGO/pGGO) & $12(0 / 12)$ \\
Pre-invasive lesion & $36(9 / 27)$ \\
Minimally invasive adenocarcinoma & \\
lesion & $29(15 / 14)$ \\
Invasive adenocarcinoma lesion &
\end{tabular}

Values are expressed as the mean \pm standard deviation (range) or $n$. GGO, ground-glass opacity; m/pGGO, mixed pure/pure GGO.

lobulation, vacuolation, obvious solid component or irregular shape of solid component (Fig. 1). Finally, the pathological results following surgery were PIL, MIA or IA. Based on these criteria, $77 \mathrm{GGO}$ nodules in 77 individuals (mean age, 53.40 \pm 11.13 years; range, 23-75 years) were selected as the study population. They included 25 males (mean age, $54.88 \pm 10.74$ years; range, $28-75$ years) and 52 females (mean age, 52.69 \pm 11.35 years; range, 23-72 years), and the mean time interval between CECT and NECT was 11 days (range, 0-81 days). Of the 77 GGO nodules, 12 GGO nodules were PIL [12 were pure GGO (pGGO) nodules], 36 were MIA [9 mixed pure GGP (mGGO) nodules and 27 were pGGO] and 29 were IA (15 mGGO and 14 pGGO). The patient characteristics are summarized in Table I.

CT examination. All enrolled individuals underwent at least one CT plain scan and one CT contrast-enhanced scan using one of these three scanners [LightSpeed VCT, GE Medical Systems, Milwaukee; Discovery CT 750 HD, GE Healthcare; GE Optima CT660(128T)], and the interval time was no more than 3 months. All CT scans were performed using the following parameters: $120 \mathrm{kVp}, 210 \mathrm{mAsec}, 0.984 / 1.375$ pitch, a reconstruction interval of $1.25 \mathrm{~mm}$ and a scan range from apex to the base of the lungs. When more than one CT plain or contrast-enhanced examination was performed, the two closest to each other were selected.

Feature extraction. All the thin-section CT images with pulmonary window (non-enhanced and contrast-enhanced) were transferred and stored as digital imaging and communications in medicine files. Nodule segmentation was performed manually. Regions of interest were drawn around the boundary of GGO nodules and the whole nodule volume was included. The images were processed using the in-house developed software coded in MATLAB (version 7.3.0) and the features were extracted automatically. Subsequently, a three-dimensional nodule was segmented and various texture features were calculated and extracted automatically. Analyzed texture features included histogram features and gray-level co-occurrence matrix (GLCM) features. Histogram features included mean attenuation, standard deviation (sd) of attenuation, skewness, kurtosis, CT attenuation values at the 10, 25, 50, 75 and 90th percentile, energy, 
Table II. Comparison of texture features between IA and PIL/MIA with non-enhanced images and contrast-enhanced images.

A, Non-enhanced CT

\begin{tabular}{|c|c|c|c|c|}
\hline Characteristics & IA & MIA or PIL & $\mathrm{t}$ & P-value \\
\hline \multicolumn{5}{|l|}{ Histogram analysis } \\
\hline Mean (HU) & $-147.49 \pm 135.86$ & $-564.56 \pm 127.55$ & -3.03 & 0.003 \\
\hline $\mathrm{Sd}(\mathrm{HU})$ & $200.38 \pm 42.38$ & $167.85 \pm 44.30$ & -3.17 & 0.002 \\
\hline Skewness & $0.51 \pm 0.54$ & $0.72 \pm 0.55$ & 1.63 & 0.108 \\
\hline Kurtosis & $0.71 \pm 1.22$ & $1.43 \pm 1.97$ & 1.96 & 0.053 \\
\hline 10th percentile (HU) & $-708.35 \pm 99.69$ & $-757.37 \pm 91.89$ & -2.20 & 0.031 \\
\hline 25th percentile $(\mathrm{HU})$ & $-609.34 \pm 125.48$ & $-680.44 \pm 106.20$ & -2.66 & 0.010 \\
\hline 50th percentile $(\mathrm{HU})$ & $-491.24 \pm 149.18$ & $-581.00 \pm 134.13$ & -2.73 & 0.008 \\
\hline 75th percentile $(\mathrm{HU})$ & $-351.65 \pm 163.59$ & $-470.46 \pm 157.30$ & -3.16 & 0.002 \\
\hline 90th percentile (HU) & $-202.75 \pm 172.55$ & $-346.26 \pm 181.87$ & -3.42 & 0.001 \\
\hline \multicolumn{5}{|l|}{ GLCM } \\
\hline Energy & $0.009 \pm 0.02$ & $0.012 \pm 0.03$ & 5.64 & $<0.001$ \\
\hline Entropy & $7.10 \pm 0.20$ & $6.69 \pm 0.35$ & -6.50 & $<0.001$ \\
\hline Correlation $\left(\mathrm{x} 10^{4}\right)$ & $3.40 \pm 1.20$ & $2.60 \pm 1.10$ & -2.71 & 0.008 \\
\hline Uniformity & $1.49 \pm 0.23$ & $1.33 \pm 0.18$ & -3.21 & 0.002 \\
\hline
\end{tabular}

B, Contrast-enhanced CT

\begin{tabular}{|c|c|c|c|c|}
\hline Characteristics & IA & MIA or PIL & $\mathrm{t}$ & P-value \\
\hline \multicolumn{5}{|l|}{ Histogram analysis } \\
\hline Mean (HU) & $-459.26 \pm 134.12$ & $-524.40 \pm 140.46$ & -2.01 & 0.049 \\
\hline $\mathrm{Sd}(\mathrm{HU})$ & $202.71 \pm 54.29$ & $171.28 \pm 55.21$ & -2.43 & 0.017 \\
\hline Skewness & $0.63 \pm 0.54$ & $0.77 \pm 0.51$ & 1.08 & 0.282 \\
\hline Kurtosis & $1.12 \pm 1.69$ & $1.45 \pm 1.50$ & 0.90 & 0.372 \\
\hline 10th percentile (HU) & $-692.91 \pm 118.39$ & $-721.70 \pm 104.50$ & -1.11 & 0.269 \\
\hline 25 th percentile $(\mathrm{HU})$ & $-597.91 \pm 134.46$ & $-639.12 \pm 123.52$ & -1.37 & 0.174 \\
\hline 50th percentile (HU) & $-482.79 \pm 142.57$ & $-543.04 \pm 145.20$ & -1.78 & 0.080 \\
\hline 75th percentile (HU) & $-344.76 \pm 152.45$ & $-432.63 \pm 169.24$ & -2.29 & 0.025 \\
\hline 90th percentile (HU) & $-186.10 \pm 170.60$ & $-303.80 \pm 197.34$ & -2.67 & 0.009 \\
\hline \multicolumn{5}{|l|}{ GLCM } \\
\hline Energy & $0.10 \pm 0.002$ & $0.12 \pm 0.003$ & 3.82 & $<0.001$ \\
\hline Entropy & $7.00 \pm 0.21$ & $6.69 \pm 0.002$ & -4.60 & $<0.001$ \\
\hline Correlation $\left(\times 10^{4}\right)$ & $3.50 \pm 0.90$ & $2.70 \pm 1.20$ & -3.11 & 0.002 \\
\hline Uniformity & $1.50 \pm 0.23$ & $1.37 \pm 0.31$ & -1.61 & 0.113 \\
\hline
\end{tabular}

IA, invasive adenocarcinoma; PIL, pre-invasive lesion; MIA, invasive adenocarcinoma; Sd, standard deviation; GLGM, grey-level co-occurrence matrix; HU, Hounsfield Units.

entropy, correlation and uniformity. GLCM features included energy, entropy, correlation and uniformity.

Statistical analysis. Differences between IA and PIL/MIA were analyzed using the independent-samples t-test for differences in histograms and GLCM features for NECT and CECT images. Furthermore, multivariate regression and receiver operating characteristic (ROC) analyses were performed to evaluate the performance of all of the significant parameters obtained using the independent-samples t-test. Statistical significance was assessed using software (SPSS version 19.0; IBM Corp.). $\mathrm{P}<0.05$ was considered to indicate a statistically significant difference.

\section{Results}

Comparison of texture features between IA and PIL/MIA. Significant differences were identified between IA and PIL/MIA with NECT images in terms of the mean and sd of attenuation, CT attenuation values at the 10, 25, 50, 75 and 
Table III. Results of receiver operating characteristic curve analysis for differentiating between IA and PIL/MIA with non-enhanced and contrast-enhanced CT images.

A, Non-enhanced CT

\begin{tabular}{lcccc}
\hline Feature & AUC & Cutoff value & Sensitivity (\%) & Specificity (\%) \\
\hline Mean (HU) & 0.708 & -612.55 & 89.7 & 50.0 \\
Sd (HU) & 0.700 & 161.17 & 86.2 & 47.9 \\
10th percentile (HU) & 0.656 & -762.73 & 72.4 & 46.2 \\
25th percentile (HU) & 0.668 & -670.67 & 69.0 & 66.7 \\
50th percentile (HU) & 0.682 & -535.53 & 69.0 & 66.7 \\
75th percentile (HU) & 0.716 & -520.38 & 82.8 & 56.2 \\
90th percentile (HU) & 0.734 & -508.67 & 85.4 & 59.2 \\
Energy & 0.839 & 0.009342 & 89.7 & 72.4 \\
Entropy & 0.859 & 6.87 & 96.6 & 66.7 \\
Correlation & 0.667 & 0.0002182 & 79.3 \\
Uniformity & 0.726 & 1.33 & 60.4 \\
\hline
\end{tabular}

B, Contrast-enhanced CT

\begin{tabular}{lcccc}
\hline Feature & AUC & Cutoff value & Sensitivity (\%) & Specificity (\%) \\
\hline Mean (HU) & 0.649 & -527.84 & 72.4 & 62.5 \\
Sd (HU) & 0.660 & 179.19 & 72.4 & 58.3 \\
75th percentile (HU) & 0.675 & -406.85 & 72.4 & 66.7 \\
90th percentile (HU) & 0.693 & -256.01 & 72.4 & 66.7 \\
Energy & 0.808 & 0.10 & 75.0 & 79.3 \\
Entropy & 0.820 & 6.85 & 82.8 & 72.9 \\
Correlation & 0.711 & 0.0002943 & 79.3 & 60.4 \\
\hline
\end{tabular}

IA, invasive adenocarcinoma; PIL, pre-invasive lesion; MIA, invasive adenocarcinoma; HU, Hounsfield Units; Sd, standard deviation; AUC, area under the curve.

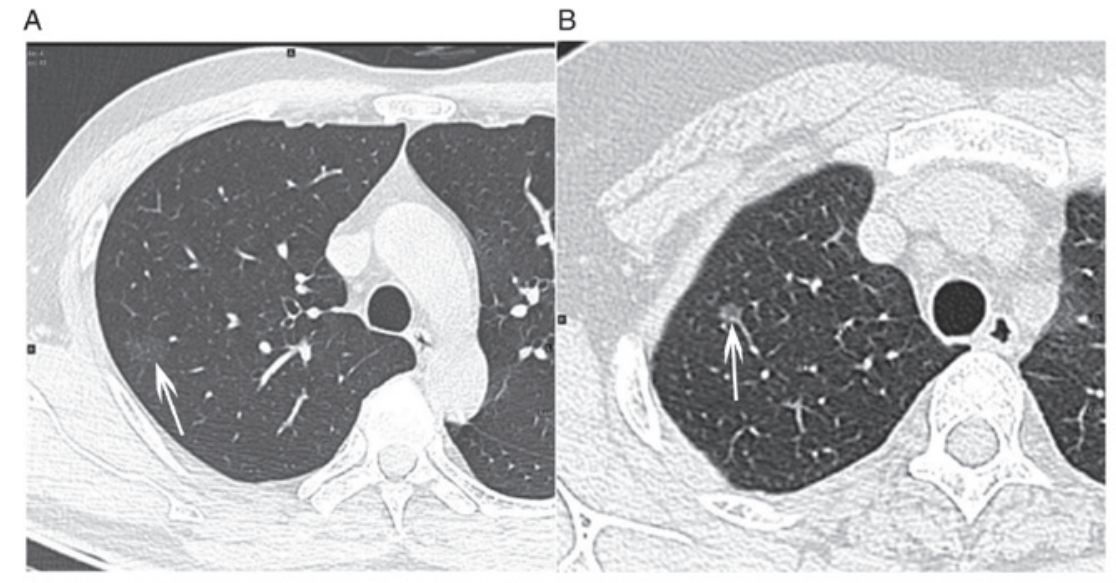

Figure 1. (A) Pure GGO nodule with regular shape, no calcification, no cavitation, no lobulation and no burr sign was present on the axial CT image (lung window) of a 54-year-old male with adenocarcinoma in situ confirmed using biopsy. (B) Mixed GGO, also with regular shape, no calcification, no cavitation, no lobulation, no burr sign and a small amount of solid component displayed on the axial CT image (lung window) of a 52-year-old female with minimally invasive lung adenocarcinoma confirmed using biopsy. White arrows indicate locations of the lesions. GGO, ground glass opacity.

90th percentile, energy, entropy, correlation and uniformity $(\mathrm{P}<0.05)$, but not for skewness $(\mathrm{P}=0.11)$ and kurtosis $(\mathrm{P}=0.06)$ (Table II). With the CECT images, mean and sd of attenuation,
CT attenuation values at the 75 and 90th percentile, energy, entropy and correlation for IA were significantly different from those for PIL/MIA, but no significant differences were 

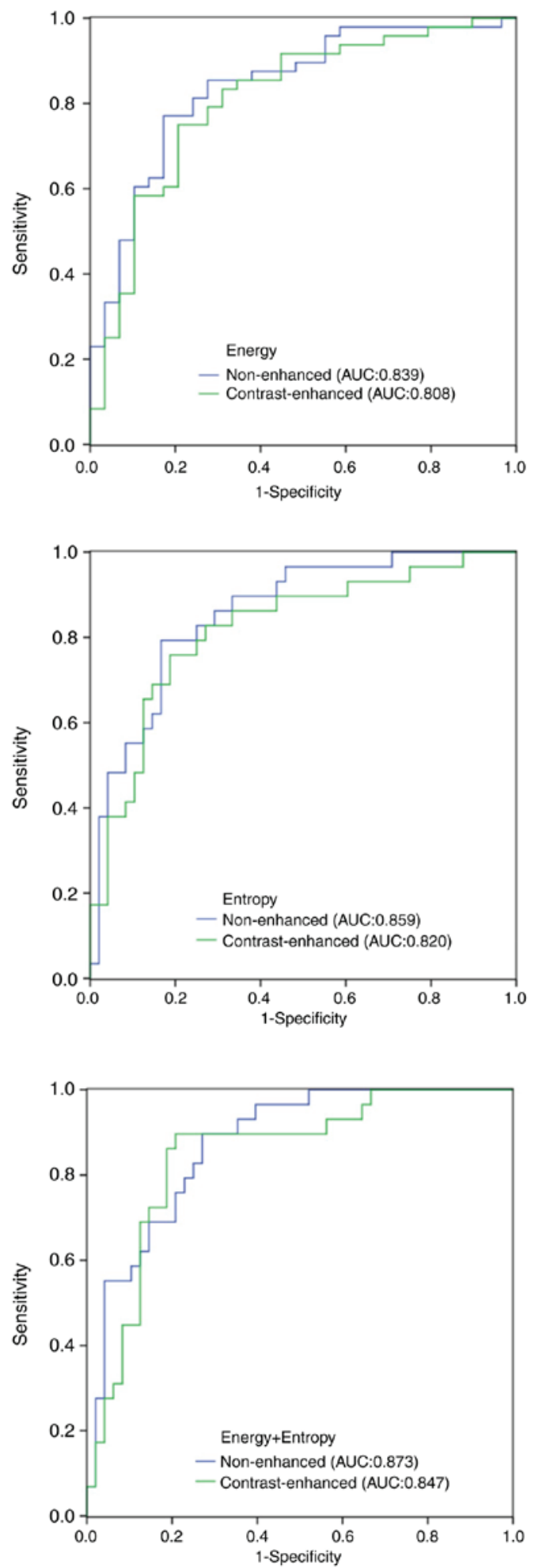

Figure 2. Results of the receiver operating characteristic curve analysis of energy and entropy in discriminating invasive adenocarcinoma from pre-invasive lesions or minimally invasive adenocarcinoma appearing as ground-glass opacity nodules. For the non-enhanced images, the AUC was 0.839 and 0.859 , respectively. For contrast-enhanced images, the AUC was 0.818 and 0.820 , respectively. When energy and entropy were used as input data at the same time, the AUC was 0.873 for non-enhanced images and 0.847 for contrast-enhanced images. AUC, area under the curve.

obtained for skewness $(\mathrm{P}=0.28)$, kurtosis $(\mathrm{P}=0.37), \mathrm{CT}$ attenuation values at the 10 th $(\mathrm{P}=0.27)$, 25th $(\mathrm{P}=0.17)$ and 50th percentile $(\mathrm{P}=0.08)$, and uniformity $(\mathrm{P}=0.11$; Table II).
Multivariate logistic regression and ROC curve analyses. In the ROC curve analysis, the area under the curve (AUC) values of all the significant parameters were obtained. For the NECT images, the AUC of the mean and Sd of attenuation, CT attenuation values at the 10, 25, 50, 75 and 90th percentile, energy, entropy, correlation and uniformity was $0.708,0.700$, $0.656,0.668,0.682,0.716,0.734,0.839,0.859,0.667$ and 0.726 , respectively. For the CECT images, the AUC of the mean and sd of attenuation, CT attenuation values at the 75 and 90th percentile, energy, entropy and correlation was $0.649,0.660$, $0.675,0.693,0.808,0.820$ and 0.711 , respectively (Table III). In the ROC analysis, when energy and entropy were used as input data at the same time, the AUC was 0.873 with NECT images and 0.847 with CECT images (Fig. 2). On CECT images, the display of a small part of GGOs near the axillary vein, subclavian vein or superior vena cava may have been interfered due to beam hardening artifacts (Fig. 3).

\section{Discussion}

The present study characterized GGO nodules using NECT and CECT texture analyses. Each of the two methods had the ability to differentiate IA from PIL/MIA. Pulmonary adenocarcinomas displaying as GGO nodules are heterogeneous at the genetic and histopathological level. Heterogeneity is a recognized feature of malignancy, reflecting areas of high cell density, necrosis, hemorrhage and myxoid change (11). Texture analysis is an important method of medical image processing that quantifies the information obtained from standard images by detecting the distribution and association of subtle pixel or voxel gray levels in the images, thus extracting numerous quantitative parameters associated with tissue heterogeneity (12).

In the present study, all PIL presented as pGGO nodules, certain MIA and IA presented as pGGO nodules and others presented as mGGO nodules. The present results were similar to those of Lee et al (13), in which most mGGOs were MIA or IA, whilst pGGOs included a variety of pathological types: A total of 20 out of $25(80 \%)$ AIS, as did one pleomorphic carcinoma and one AAH. Several studies indicated that GGO nodules with spiculation, lobulation, or vacuolation sign are suggestive of IA $(14,15)$. GGO nodules containing solid components or irregular morphology of solid components accurately suggested IA. To better solve practical clinical problems, cases with obvious signs of malignancy, including speculation, lobulation, vacuolation, obvious solid component or irregular shape of the solid component, were removed. Eventually, patients with pGGO or mGGO nodules containing small amounts and regular morphology of solid components were enrolled in the present study.

Numerous studies have confirmed the application of texture analysis in diagnosing pulmonary nodules. Lee et al (16) demonstrated that the texture analysis of par-solid GGO nodules has the potential to improve the differentiation of transient from persistent par-solid GGO nodules when used in addition to the clinical and CT feature analysis. In the ROC analysis, when clinical and CT features were used as input data, the AUC was 0.79, and when CT texture analysis features was used as input data, the AUC was 0.81. However, when the features of clinical, CT imaging and CT texture analysis were used as input data, the AUC was calculated to be 0.93. A total 


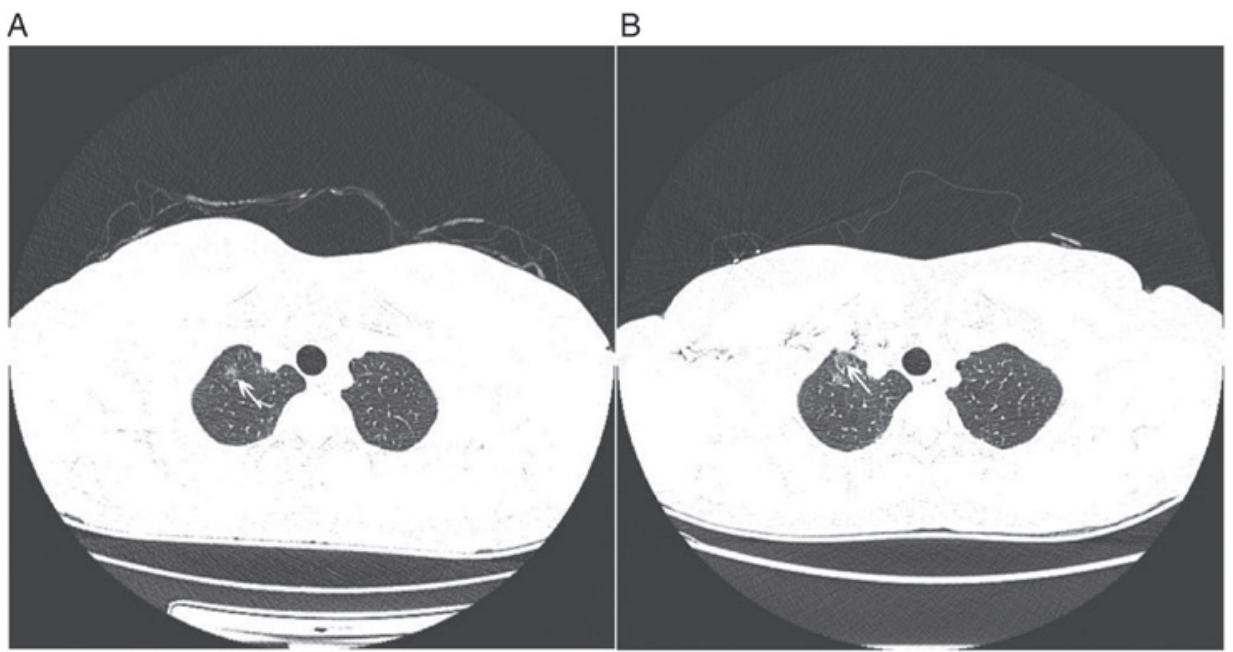

Figure 3. (A) A ground-glass opacity nodule in the upper lobe of the right lung clearly displayed on non-enhanced CT images. (B) However, on contrast-enhanced $\mathrm{CT}$, the same nodule only displayed vaguely due to an obvious beam-hardening artifact.

of three studies performed a texture analysis of CT imaging to help distinguish the infiltration degree of pulmonary adenocarcinoma appearing as GGO nodules with no or little solid component $(17,18)$, which were consistent with the present study. Li et al (19) divided the samples into three groups, namely PIL, MIA and IA. Son et al (17) and Chae et al (18) divided the samples into two groups, namely PIL/MIA and IA, as in the present study. The 5-year disease-free survival rate of patients with PIL and MIA is $100 \%$ after receiving segmentectomy of the lungs $(2,3)$. However, for IA, lobectomy is the major method and the prognosis is relatively poor $(2,3)$. Therefore, early distinction of IA from PIL or MIA is critical for the selection of the surgical procedure and improvement of prognosis. Therefore, stratification into two groups not only makes the experimental design simple, but also is able to better solve practical clinical problems. The three studies used NECT images for texture analysis, while certain other studies on pulmonary nodules used CECT images $(20,21)$. However, none of them clearly explained why the NECT or CECT images were chosen. The present study investigated and compared the value of NECT and CECT texture analysis in differentiating IA from PIL/MIA. Son et al (17) concluded that the 75th percentile CT attenuation and entropy were significant independent factors to predict IA. Chae et al (18) concluded that the mass and kurtosis were significant independent factors to predict IA.

In the present study, a total of 13 texture analysis parameters were selected. Of these, 11 parameters exhibited a statistically significant difference with NECT images between IA and MIA/PIL, whilst smaller energy and higher entropy were significant differentiators of IA from MIA/PIL. Furthermore, 8 parameters exhibited a statistically significant difference with CECT images betweenIA and MIA/PIL and similarly, the smaller energy and higher entropy were significant differentiators of IA from MIA/PIL. Several studies have suggested that a higher entropy is associated with malignancy in lung cancer $(17,20)$, liver cirrhosis (22) and adnexal neoplasms $(23,24)$. Entropy is the characteristic parameter to measure the randomness of the gray-level distribution, which represents the complexity of the image texture. A more complex the image texture is associated with a higher entropy value (25). Energy reflects the uniformity of gray distribution and coarseness of texture. The more uniform the image, the higher the energy. Increased infiltration of lung adenocarcinoma is accompanied by changes in cell permeability, abnormal angiogenesis, viscous liquefaction and necrosis, leading to heterogeneity of the tumor. Therefore, IA is characterized by mixed and heterogeneous components in pathology; it cannot be accurately distinguished by intuitive CT image features, but it may be detected and quantified by texture analysis. The present study indicated that the energy of IA was smaller than that of PIL/MIA and the entropy of IA was larger than that of PIL/MIA. Furthermore, it indicated that the gray distribution of IA was relatively heterogeneous, while the gray distribution of PIL/MIA was more uniform.

It was initially assumed that skewness or kurtosis may help differentiate IA from PIL/MIA, as indicated in numerous previous studies $(15,19,26,27)$. However, the present results did not confirm this, as no significant difference was obtained. It was presumed that the variation of the histogram graphs of IA, PIL and MIA is too high to provide a distinction between them.

Several studies confirmed the use of the whole nodule, segmented slice-by-slice imaging of the lesion on thin-section CT images until the entire GGO had been covered instead of using the largest diameter of the GGO to extract features $(25,28,29)$, despite the use of the largest diameter of the GGO being more time-efficient. In addition, the selection of the largest diameter of the GGO may vary among different radiologists. Therefore, in the present study, the three-dimensional imaging of lesions was extracted for texture analysis.

In the present study, more parameters from NECT images exhibited statistically significant differences between IA and PIL/MIA compared with those from CECT images. The diagnostic efficiency of energy and entropy from NECT images were slightly higher than those from CECT images, although they had an excellent performance. The reasons may be as follows: First, CECT images obtained with the use of iodine contrast agent provided insight into lesion heterogeneity predominantly linked to the presence of areas 
with different vascularization. However, texture analysis may reflect cellular distribution on NECT images. Furthermore, on CECT scanning, a high concentration of iodine contrast media in the axillary vein, subclavian vein and superior vena cava may produce an obvious beam hardening artifact and may interfere with the display of GGO lesions that happen to be nearby. However, CECT imaging has an essential role in the diagnosis of GGO nodules. It may provide the blood supply of the lesion and judge whether the pulmonary vessels are invaded. Therefore, for GGO nodules, CECT scanning is necessary when deciding to perform surgical resection. In the process of regular review and comparative observation of GGO, NECT imaging may be the major method. On this basis, NECT images were simpler to obtain and no contrast agent was required. Thus, for CT texture analysis only, NECT texture analysis may be a better choice.

The present study had certain limitations. First, it had a retrospective design, leading to potential selection bias. Furthermore, the sample size was relatively small. In addition, the CT examinations were not performed with the same CT machine, but three different machines, resulting in variability of the $\mathrm{CT}$ value and, to a certain extent, affecting the accuracy of the texture analysis. As another limitation, the boundary of GGO nodules was manually segmented by the radiologist and may have been influenced by the subjective trend or bias of the observer, particularly for GGO nodules with a fuzzy boundary. Finally, in addition to pulmonary adenocarcinoma, benign lesions, including focal inflammation, edema or hemorrhage, may also display as GGO. The present study only included GGO confirmed as pulmonary adenocarcinoma. Therefore, a more extensive study will be the next research focus.

In conclusion, lower energy and higher entropy are significant differentiators of IA from PIA/MIA in pulmonary adenocarcinoma displaying as GGO nodules. NECT and CECT texture analyses have the potential to differentiate IA from PIA/MIA; however, for texture analysis only, NECT texture analysis may be a preferred choice.

\section{Acknowledgements}

Not applicable.

\section{Funding}

The present study was supported by funding from Subei People's Hospital (grant no. yzucms201804; Yangzhou, China).

\section{Availability of data and materials}

The datasets used and/or analyzed during the present study are available from the corresponding author on reasonable request.

\section{Authors' contributions}

JY and YL were involved in the conception, design, definition of intellectual content, literature search, data acquisition, data analysis and manuscript preparation. JL and JHC provided assistance with data acquisition, data analysis and statistical analysis. JC and MC performed the literature search, data acquisition and manuscript editing. All authors read and approved the content of the manuscript.

\section{Ethics approval and consent to participate}

The present retrospective study was approved by the institutional review board of Yangzhou University Clinical College Subei People's Hospital (approval no. 2018KY-029; Yangzhou. China) with a waiver of informed consent.

\section{Patient consent for publication}

Not applicable.

\section{Competing interests}

All authors declare that they have no competing interests.

\section{References}

1. Travis WD, Brambilla E, Noguchi M, Nicholson AG, Geisinger KR, Yatabe Y, Beer DG, Powell CA, Riely GJ, Van Schil PE, et al: International association for the study of lung cancer/american thoracic society/european respiratory society international multidisciplinary classification of lung adenocarcinoma. J Thorac Oncol 6: 244-285, 2011.

2. Borczuk AC, Qian F, Kazeros A, Eleazar J, Assaad A, Sonett JR, Ginsburg M, Gorenstein L and Powell CA: Invasive size is an independent predictor of survival in pulmonary adenocarcinoma. Am J Surg Pathol 33: 462-469, 2009.

3. Zhang J, Wu J, Tan Q, Zhu L and Gao W: Why do pathological stage IA lung adenocarcinomas vary from prognosis?: A clinicopathologic study of 176 patients with pathological stage IA lung adenocarcinoma based on the IASLC/ATS/ERS classification. J Thorac Oncol 8: 1196-1202, 2013.

4. Kim HY,Shim YM,Lee KS, Han J, Yi CA and Kim YK: Persistent pulmonary nodular ground-glass opacity at thin-section CT: Histopathologic comparisons. Radiology 245: 267-275, 2007.

5. Lee SM, Park CM, Goo JM, Lee HJ, Wi JY and Kang CH: Invasive pulmonary adenocarcinomas versus preinvasive lesions appearing as ground-glass nodules: Differentiation by using CT features. Radiology 268: 265-273, 2013.

6. Lee HJ, Goo JM, Lee CH, Yoo CG, Kim YT and Im JG: Nodular ground-glass opacities on thin-section CT: Size change during follow-up and pathological results. Korean J Radiol 8: 22-31, 2007.

7. Park CM, Goo JM, Lee HJ, Lee CH, Chun EJ and Im JG: Nodular ground-glass opacity at thin-section CT: Histologic correlation and evaluation of change at follow-up. Radiographics 27: 391-408, 2007.

8. Lee HY and Lee KS: Ground-glass opacity nodules: Histopathology, imaging evaluation, and clinical implications. J Thorac Imaging 26: 106-118, 2011.

9. Travis WD, Garg K, Franklin WA, Wistuba II, Sabloff B, Noguchi M, Kakinuma R, Zakowski M, Ginsberg M, Padera R, et al: Evolving concepts in the pathology and computed tomography imaging of lung adenocarcinoma and bronchioloalveolar carcinoma. J Clin Oncol 23: 3279-3287, 2005.

10. Lim HJ, Ahn S, Lee KS, Han J, Shim YM, Woo S, Kim JH, Yie M, Lee HY and Yi CA: Persistent pure ground-glass opacity lung nodules $\geq 10 \mathrm{~mm}$ in diameter at CT scan: Histopathologic comparisons and prognostic implications. Chest 144: 1291-1299, 2013.

11. Nelson DA, Tan TT, Rabson AB, Anderson D, Degenhardt K and White E: Hypoxia and defective apoptosis drive genomic instability and tumorigenesis. Genes Dev 18: 2095-2107, 2004.

12. Ganeshan B and Miles KA: Quantifying tumour heterogeneity with CT. Cancer Imaging 13: 140-149, 2013.

13. Lee SW, Leem CS, Kim TJ, Lee KW, Chung JH, Jheon S, Lee JH and Lee CT: The long-term course of ground-glass opacities detected on thin-section computed tomography. Respir Med 107: 904-910, 2013.

14. Lee HJ, Goo JM, Lee CH, Park CM, Kim KG, Park EA and Lee HY: Predictive CT findings of malignancy in ground-glass nodules on thin-section chest CT: The effects on radiologist performance. Eur Radiol 19: 552-560, 2009. 
15. Aoki T, Tomoda Y, Watanabe H, Nakata H, Kasai T, Hashimoto H, Kodate M, Osaki T and Yasumoto K: Peripheral lung adenocarcinoma: Correlation of thin-section CT findings with histologic prognostic factors and survival. Radiology 220: 803-809, 2001.

16. Lee SH, Lee SM, Goo JM, Kim KG, Kim YJ and Park CM: Usefulness of texture analysis in differentiating transient from persistent part-solid nodules(PSNs): A retrospective study. PLoS One 9: e85167, 2014

17. Son JY, Lee HY, Lee KS, Kim JH, Han J, Jeong JY, Kwon OJ and Shim YM: Quantitative CT analysis of pulmonary ground-glass opacity nodules for the distinction of invasive adenocarcinoma from pre-invasive or minimally invasive adenocarcinoma. PLoS One 9: e104066, 2014.

18. Chae HD, Park CM, Park SJ, Lee SM, Kim KG and Goo JM Computerized texture analysis of persistent part-solid ground-glass nodules: Differentiation of preinvasive lesions from invasive pulmonary adenocarcinomas. Radiology 273: 285-293, 2014.

19. Li W, Wang X, Zhang Y, Li X, Li Q and Ye Z: Radiomic analysis of pulmonary ground-glass opacity nodules for distinction of preinvasive lesions, invasive pulmonary adenocarcinoma and minimally invasive adenocarcinoma based on quantitative texture analysis of CT. Chin J Cancer Res 30: 415-424, 2018

20. Liu Y, Liu S, Qu F, Li Q, Cheng R and Ye Z: Tumor heterogeneity assessed by texture analysis on contrast-enhanced CT in lung adenocarcinoma: Association with pathologic grade. Oncotarget 8: 53664-53674, 2017.

21. Suo S, Cheng J, Cao M, Lu Q, Yin Y, Xu J and Wu H: Assessment of heterogeneity difference between edge and core by using texture analysis: Differentiation of malignant from inflammatory pulmonary nodules and masses. Acad Radiol 23: 1115-1122, 2016.

22. Fujimoto K, Tonan T, Azuma S, Kage M, Nakashima O, Johkoh T, Hayabuchi N, Okuda K, Kawaguchi T, Sata M and Qayyum A: Evaluation of the mean and entropy of apparent diffusion coefficient values in chronic hepatitis C: Correlation with pathologic fibrosis stage and inflammatory activity grade. Radiology 258: 739-748, 2011.
23. Kierans AS, Bennett GL, Mussi TC, Babb JS, Rusinek H, Melamed J and Rosenkrantz AB: Characterization of malignancy of adnexal lesions using ADC entropy: Comparison with mean ADC and qualitative DWI assessment. J Magn Reson Imaging 37: 164-171, 2013.

24. Cao MQ, Suo ST, Zhang XB, Zhong YC, Zhuang ZG, Cheng JJ, Chi JC and Xu JR: Entropy of T2-weighted imaging combined with apparent diffusion coefficient in prediction of uterine leiomyoma volume response after uterine artery embolization. Acad Radiol 21: 437-444, 2014.

25. Ganeshan B, Miles KA, Young RC and Chatwin CR: Hepatic entropy and uniformity: Additional parameters that can potentially increase the effectiveness of contrast enhancement during abdominal CT. Clin Radiol 62: 761-768, 2007.

26. Chandarana H, Rosenkrantz AB, Mussi TC, Kim S, Ahmad AA, Raj SD, McMenamy J, Melamed J, Babb JS, Kiefer B and Kiraly AP: Histogram analysis of whole-lesion enhancement in differentiating clear cell from papillary subtype of renal cell cancer. Radiology 265: 790-798, 2012.

27. Wang S, Kim S, Zhang Y, Wang L, Lee EB, Syre P, Poptani H, Melhem ER and Lee JY: Determination of grade and subtype of meningiomas by using histogram analysis of diffusion-tensor imaging metrics. Radiology 262: 584-592, 2012.

28. Ganeshan B, Abaleke S, Young RC, Chatwin CR and Miles KA: Texture analysis of non-small cell lung cancer on unenhanced computed tomography: Initial evidence for a relationship with tumour glucose metabolism and stage. Cancer Imaging 10: 137-143, 2010.

29. Ravanelli M, Farina D, Morassi M, Roca E, Cavalleri G, Tassi G and Maroldi R: Texture analysis of advanced non-small cell lung cancer (NSCLC) on contrast-enhanced computed tomography: Prediction of the response to the first-line chemotherapy. Eur Radiol 23: 3450-3455, 2013.

This work is licensed under a Creative Commons Attribution-NonCommercial-NoDerivatives 4.0 International (CC BY-NC-ND 4.0) License. 